\title{
Shape Analysis of Vestibular Systems in Adolescent Idiopathic Scoliosis Using Geodesic Spectra
}

\author{
Wei Zeng ${ }^{1,4}$, Lok Ming Lui ${ }^{2}$, Lin $\mathrm{Shi}^{3}$, Defeng Wang ${ }^{3}$, Winnie C.W. Chu ${ }^{3}$, \\ Jack C.Y. Cheng ${ }^{3}$, Jing Hua ${ }^{1}$, Shing-Tung $\mathrm{Yau}^{2}$, and Xianfeng Gu ${ }^{4}$ \\ ${ }^{1}$ Wayne State University, Detroit, MI 48202, USA \\ ${ }^{2}$ Harvard University, Cambridge, MA 02138, USA \\ 3 The Chinese University of Hong Kong, Hong Kong, China \\ ${ }^{4}$ Stony Brook University, Stony Brook, NY 11794, USA
}

\begin{abstract}
Adolescent Idiopathic Scoliosis (AIS) characterized by the $3 \mathrm{D}$ spine deformity affects about $4 \%$ schoolchildren worldwide. One of the prominent theories of the etiopathogenesis of AIS was proposed to be the poor postural balance control due to the impaired vestibular function. Thus, the morphometry of the vestibular system (VS) is of great importance for studying AIS. The VS is a genus-3 structure situated in the inner ear and consists of three semicircular canals lying perpendicular to each other. The high-genus topology of the surface poses great challenge for shape analysis. In this work, we propose an effective method to analyze shapes of high-genus surfaces by considering their geodesic spectra. The key is to compute the canonical hyperbolic geodesic loops of the surface, using the Ricci flow method. The Fuchsian group generators are then computed which can be used to determine the geodesic spectra. The geodesic spectra effectively measure shape differences between highgenus surfaces up to the hyperbolic isometry. We applied the proposed algorithm to the VS of 12 normal and 15 AIS subjects. Experimental results show the effectiveness of our algorithm and reveal statistical shape difference in the VS between right-thoracic AIS and normal subjects.
\end{abstract}

\section{Introduction}

Adolescent Idiopathic Scoliosis (AIS) is a 3D spinal deformity which affects about $4 \%$ schoolchildren worldwide. It is believed to be a multi-factorial disease. The right-thoracic curve is the most typical type of AIS. The current treatment of AIS is still unsatisfactory because the etiopathogenesis remains unclear. AIS patients had a significantly higher mean body sway compared with healthy controls when their visual and somatosensory systems were simultaneously challenged 3 . It has been proposed that the altered processing of vestibular information might contribute to the etiology of AIS [11]. Based on the findings of abnormal postural balance control in AIS 391121, one of the prominent theories of the etiopathogenesis of AIS was proposed to be the poor postural balance control

T. Jiang et al. (Eds.): MICCAI 2010, Part III, LNCS 6363, pp. 538 546, 2010.
(C) Springer-Verlag Berlin Heidelberg 2010 
due to the impaired vestibular function [22]. The vestibular system (VS) is responsible for perception of head movements and sending postural signals to the brain. The important role of the vestibular system in the development of idiopathic scoliosis was manifested by an animal study using the frog model [14]. Recently, some preliminary results have also revealed the statistical difference in global morphology of the VS between right-thoracic AIS and normal controls 20. The shape of the central contour of each canal for the VS surfaces has also been studied [2]. However, the complete geometry of the surface has not been fully analyzed. Thus, the morphometry of the VS is of great importance for understanding AIS. The VS is a genus-3 structure situated in the inner ear and consists of three semicircular canals lying perpendicular to each other, see Figure 1 (a). Because of the high-genus topology of the VS, it poses great challenge for shape analysis. This motivates us to propose a stable algorithm to study the VS systematically by making full use of rigorous structural features.

A lot of works have been done on shape analysis for anatomical structures. For example, Dale et al. 6] proposed a surface-based methods for studying the structural features of brain; Ashburner et al. [1] proposed a deformation-based morphometry for studying the $2 \mathrm{D}$ or $3 \mathrm{D}$ local displacement vector fields in brain volume or shape; Pizer et al. 15] proposed to measure shape differences by medial representations (M-reps); Davies et al. [7] developed an algorithm for shape discrimination in the hippocampus using the minimum description length (MDL) models; Chung et al. 5] proposed a tensor-based morphometry for examining spatial derivatives of deformation maps using spherical harmonic representation; Reuter et al. 17 16] presented Laplace-Beltrami spectra as shape DNA for statistical shape analysis. These methods provide a good way for analyzing anatomical shapes, but they generally cannot deal with high-genus structures.

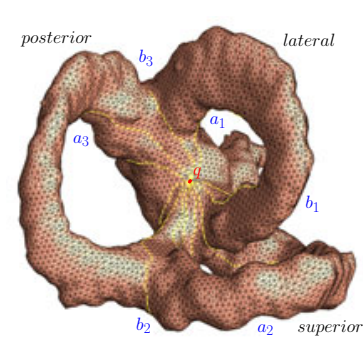

(a) fundamental group

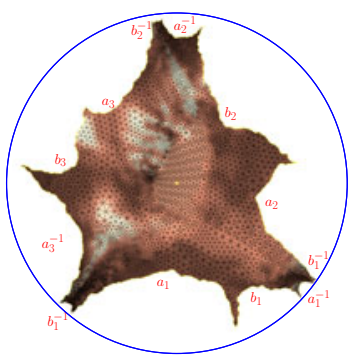

(b) fundament domain

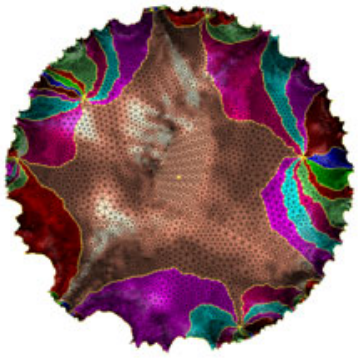

(c) portion of UCS

Fig. 1. Computing the hyperbolic metric for a vestibular system (VS) surface. (a) The VS is of genus-3 and consists of 3 semicircular canals lying perpendicular to each other: the lateral canal with $\left(a_{1}, b_{1}\right)$, the superior canal with $\left(a_{2}, b_{2}\right)$ and the posterior canal with $\left(a_{3}, b_{3}\right)$, where $\left\{a_{1}, b_{1}, a_{2}, b_{2}, a_{3}, b_{3}\right\}$ are the homotopy group generators. (b) The fundamental domain embedded in the hyperbolic space, the Poincare disk. (c) The finite portion of the universal covering space (UCS). Different fundamental domains are encoded in different colors. 
In this work, we propose an effective algorithm for shape analysis of high-genus surfaces by considering their geodesic spectra. Under the hyperbolic metric, one can find the shortest geodesic loop in each homotopy class of the surface, and the lengths of such geodesic loops form the geodesic spectrum. The geodesic spectrum determines the structure of a high-genus surface up to hyperbolic isometry. To compute the geodesic spectrum, the basic idea is to compute the hyperbolic metric using the Ricci flow method. Ricci flow was first introduced by Hamilton [10] and later it was generalized to the discrete case [4. Zeng et al. 23] applied the surface Ricci flow method to study general 3D shape matching and registration. Recently, Jin et al. [13] introduced the Teichmüller shape space to index and compare general surfaces with various topologies, geometries and resolutions. After the hyperbolic metric is computed, we can compute the Fuchsian group generators which can be used to determine the geodesic spectrum. The method is rigorous and practical. Experimental results on 12 normal and 15 AIS subjects show the effectiveness of our algorithm and reveal statistical shape difference in the VS between the normal and unhealthy groups.

\section{Theoretic Background}

This section briefly introduces the background knowledge of conformal geometry, and the basic concepts of algebraic topology and hyperbolic geometry. For more details, we refer readers to the classical textbooks [18] and [19].

\subsection{Fundamental Group and Universal Covering Space}

Let $S$ be a topological surface with a base point $q$. All loops through $q$ are classified by homotopic relations. All homotopy equivalence classes form the homotopy group or fundamental group $\pi_{1}(S, q)$, where the product is defined as the concatenation of two loops through their common base point. For a genus $g$ closed surface, one can find canonical homotopy group generators $\left\{a_{1}, b_{1}, a_{2}, b_{2}, \cdots, a_{g}, b_{g}\right\}$, such that $a_{k}$ only intersects $b_{k}$ once. Then the fundamental group is represented as $<a_{1}, b_{1}, a_{2}, b_{2}, \cdots, a_{g}, b_{g} \mid a_{1} b_{1} a_{1}^{-1} b_{1}^{-1} a_{2} b_{2} a_{2}^{-1} b_{2}^{-1} \cdots a_{g} b_{g} a_{g}^{-1} b_{g}^{-1}=e>$.

A covering space of $S$ is a space $\tilde{S}$ together with a continuous surjective map $h: \tilde{S} \rightarrow S$, such that for every $p \in S$ there exists an open neighborhood $U$ of $p, h^{-1}(U)$ is a disjoint union of open sets in $\tilde{S}$, each of which is mapped homeomorphically onto $U$ by $h$. The map $h$ is called the covering map. The universal covering space (UCS) is simply connected. A deck transformation of a cover $h: \tilde{S} \rightarrow S$ is a homeomorphism $f: \tilde{S} \rightarrow \tilde{S}$ such that $h \circ f=h$. All deck transformations form the so-called deck transformation group. A fundamental domain of $S$ is a simply connected domain, which intersects each orbit of the deck transformation group only once. Figure 1 shows a fundamental domain and a finite portion of the universal covering space of a genus- 3 surface. The canonical homotopy group basis are computed using the algorithm in [8]. 


\subsection{Hyperbolic Uniformization and Ricci Flow}

Two Riemannian metrics are conformal, if they differ by a scalar function. If any surface admits a conformal Riemannian metric of constant Gaussian curvature, such metric is called the uniformization metric. The uniformization metric for a genus $g>1$ surface induces -1 Gaussian curvature [10, which is called the hyperbolic metric of the surface. The hyperbolic metric can be computed by the Ricci flow method. Intuitively, a surface Ricci flow is a process to deform the Riemannian metric of the surface, in proportion to Gaussian curvatures, such that the curvature evolves in the same manner as heat diffusion:

$$
\frac{d g_{i j}}{d t}=-2 K g_{i j}+\frac{\chi(S)}{A},
$$

where $K$ is the Gaussian curvature induced by the metric $\mathbf{g}(t), A$ is the area of the surface. For closed surfaces with non-positive Euler numbers $\chi$, Hamilton proved the convergence of Ricci flow in [10].

The universal covering space of $S$ with the hyperbolic metric can be isometrically embedded in the hyperbolic space $\mathbb{H}^{2}$, here we use Poincaré disk model. All the deck transformations are Möbius transformation, called Fuchsian transformation. The deck transformation group is called the Fuchsian group of $S$.

\subsection{Geodesic Spectrum}

Each homotopy class has a unique closed geodesic under the hyperbolic metric. The lengths of all such geodesics form the geodesic spectrum of the surface. Geodesic spectrum determines the hyperbolic metric completely.

Theorem 1 (Geodesic Spectrum). Suppose $\left(S_{1}, \mathbf{g}_{1}\right)$ and $\left(S_{2}, \mathbf{g}_{2}\right)$ are surfaces with hyperbolic metrics. Let $f: S_{1} \rightarrow S_{2}$ be a diffeomorphism, such that for each homotopy class $[\gamma] \in \pi_{1}\left(S_{1}, p\right)$, it is mapped to a homotopy class $[f(\gamma)] \in \pi_{1}\left(S_{2}, f(p)\right)$. If the length of the geodesic in $[\gamma]$ equals to the length of the geodesic in $[f(\gamma)]$, for all homotopy classes, then $f$ is an isometry.

Each homotopy class corresponds to a unique Fuchsian transformation. The length of the unique geodesic in the class can be explicitly computed by the corresponding Fuchsian transformation. If two surfaces are conformal equivalent, $f:\left(S_{1}, \mathbf{g}_{1}\right) \rightarrow$ $\left(S_{2}, \mathbf{g}_{2}\right)$ is the conformal mapping. Then under their uniformization hyperbolic metrics, $f:\left(S_{1}, \tilde{\mathbf{g}}_{1}\right) \rightarrow\left(S_{2}, \tilde{\mathbf{g}}_{2}\right)$ is an isometry. Therefore, the two surfaces have the same hyperbolic geodesic spectrum. Geodesic spectrum is the key to measure the similarity of the conformal structures of the high-genus surfaces.

In the above, the notations $g, \mathbf{g}$, and $g_{i j}$ are used to denote the concepts of surface genus, Riemannian metric and component of metric tensor, respectively.

\section{Algorithms}

The main goal of our algorithm is to compute the hyperbolic geodesic spectrum of a high-genus closed surface for shape analysis. The pipeline is as follows: 1) canonical homotopy group generators [ $[$, 2) hyperbolic metric with Ricci flow, 3) Fuchsian group generators, and 4) geodesic spectrum. 
Discrete Hyperbolic Ricci Flow. The computation of the hyperbolic metric on a triangular mesh $M$ is based on the discrete hyperbolic Ricci flow. We will briefly describe the algorithm on triangular mesh. Details can be found in [4] and $[12$.

1. Assign a circle at vertex $v_{i}$ with radius $r_{i}$; For each edge $\left[v_{i}, v_{j}\right]$, two circles intersect at an angle $\phi_{i j}$, called edge weight.

2. The edge length $l_{i j}$ of $\left[v_{i}, v_{j}\right]$ is determined by the hyperbolic cosine law,

$$
\cosh l_{i j}=\cosh r_{i} \cosh r_{j}+\sinh r_{i} \sinh r_{j} \cos \phi_{i j} .
$$

3. The angle $\theta_{i}^{j k}$, related to each corner ${ }_{i} \angle_{j}^{k}$, is determined by the current edge lengths with the inverse hyperbolic cosine law.

4. Compute the discrete Gaussian curvature $K_{i}$ of each vertex $v_{i}$ :

$$
K_{i}=\left\{\begin{array}{c}
2 \pi-\sum_{f_{i j k} \in F} \theta_{i}^{j k}, \text { interior vertex } \\
\pi-\sum_{f_{i j k} \in F} \theta_{i}^{j k}, \text { boundary vertex }
\end{array}\right.
$$

where $\theta_{i}^{j k}$ represents the corner angle attached to vertex $v_{i}$ in the face $f_{i j k}$.

5. Update the radius $r_{i}$ of each vertex $v_{i}: r_{i}=r_{i}-\epsilon K_{i} \sinh r_{i}$.

6. Repeat the step 2 through 5 , until $\left\|K_{i}\right\|$ of all vertices are less than the user-specified error tolerance.

Computing Fuchsian Group Generators. The Fuchsian group generators are obtained in the following way:

1. Slice $M$ open along a set of canonical homology basis $a_{1}, b_{1}, a_{1}^{-1}, b_{1}^{-1}, a_{2}$, $b_{2}, a_{2}^{-1}, b_{2}^{-1}$ to form the fundamental domain $\bar{M}$. See Figure 1(a).

2. Embed the fundamental domain of $M$ into Poincaré disk with boundary segments $\partial \bar{M}=a_{1} b_{1} a_{1}^{-1} b_{1}^{-1} a_{3} b_{3} a_{3}^{-1} b_{3}^{-1}$. See Figure 1(b).

3. Compute the unique Möbius transformation (hyperbolic rigid motion) that maps $a_{i}$ to $a_{i}^{-1}$ on $\bar{M}$, which is $\beta_{i}$; compute $\alpha_{j}$ which maps $b_{j}^{-1}$ to $b_{j}$.

Then we get the canonical Fuchsian group generators $\left\{\alpha_{1}, \beta_{1}, \alpha_{2}, \beta_{2}, \alpha_{3}, \beta_{3}\right\}$. Figure 1(c) shows a finite portion of the universal covering space of a genus-3 VS surface embedded in the Poincaré disk. Different fundamental domains are encoded by different colors.

Computing Geodesic Spectrum. The fundamental group generators are $\left\{a_{1}\right.$, $\left.b_{1}, a_{2}, b_{2}, a_{3}, b_{3}\right\}$. Any homotopy class is represented as a word in $\pi_{1}(S, q)$, $w=\omega_{1} \omega_{2} \cdots \omega_{n}$, the corresponding Fuchsian transformation is given by $\tau=$ $\tau_{1} \tau_{2} \cdots \tau_{n}$, where $\omega_{j}$ is replaced by $\tau_{j}$. If $\omega_{j}$ equals $a_{k}^{ \pm 1}$ (or $b_{k}^{ \pm 1}$ ), then $\tau_{j}$ is $\alpha_{k}^{ \pm 1}$ (or $\beta_{k}^{ \pm 1}$ ). Then we use the upper half plane model of $\mathbb{H}^{2} \cdot \tau$ is the product of the matrices of $\tau_{j}$. The length of the unique geodesic loop in the class of $w$ is given by the trace of the matrix representation of $\tau$,

$$
2 \cosh \left(\frac{l}{2}\right)=\operatorname{trace}(\tau)
$$

Therefore, the geodesic spectrum can be efficiently computed in an explicit form. Figure 2 visualizes the computing process of the geodesic loops for canonical homotopy group generators. 


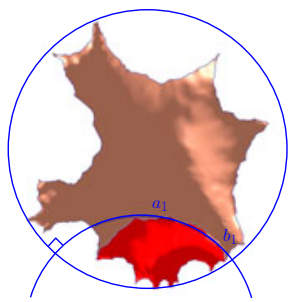

(a)

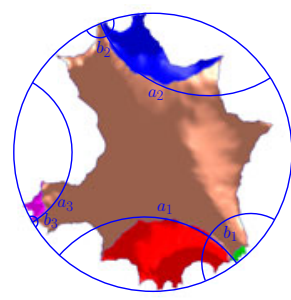

(b)

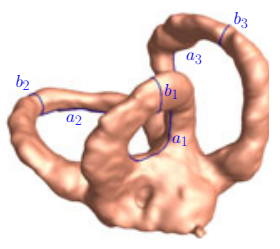

(c)

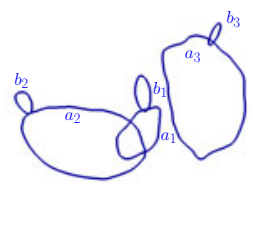

(d)

Fig. 2. Computing the geodesic loops for each homotopy class of a VS surface. (a) The unique geodesic loop in the homotopy class $\left[a_{1}\right]$ is the axis of the Möbius transformation $\alpha_{1}$ on UCS, which is a circular arc orthogonal to the unit circle on the Poincaré disk. (b) All the geodesics loops form the homotopy group basis on the Poincaré disk. (c) The geodesic loops on the original surface. (d) The geodesic loops in $\mathbb{R}^{3}$.

\section{Experiments}

Subjects and image acquisition. 15 girls with right-thoracic AIS (mean age 15 years old; mean Cobb's angle 27.27 degrees) and 12 age-matched healthy girls participated in this study. All participants in this study underwent a T2-weighted MRI scanning of the inner ear using 1.5T MR Scanner (Sonata, Siemens, Erlangen, Germany) with a quadrature head coil. High-spatial resolution anatomic imaging was performed with a 3D constructive interference steady state (CISS) sequence with $\mathrm{TR}=11.94 \mathrm{msec}, \mathrm{TE}=5.97 \mathrm{msec}$, flip angle $=70$ degrees, $\mathrm{FOV}=$ $130 \mathrm{~mm}$, slice thickness $=1 \mathrm{~mm}$, no gap, matrix $=320 \times 288$, number of excitation $=1$. The sequence yielded high quality $\mathrm{T} 2$-weighted images with the voxel size of $0.5 \times 0.4 \times 1.0 \mathrm{~mm}^{3}$. After on-site image interpolation, the voxel size was adjusted to $0.2 \times 0.2 \times 1.0 \mathrm{~mm}^{3}$.

Segmentation of the vestibular system. 3D segmentation of vestibular system from T2-MRI in this study was achieved by a tailor-made pipeline proposed in [20. Triangle mesh of the vestibular system was constructed using the marching cube algorithm. As it was found that the left-side vestibular system is abnormal in AIS compared to normal controls, in this study, our analysis is focused on the left-side vestibular system.

Computation of geodesic spectrum. The geodesic spectrums is intrinsic to the surface geometry and invariant to the triangulation resolution of the mesh. The algorithms are tested on a laptop with 2.0GHz CPU and 3.0GB RAM. The genus3 VS surfaces are with $50 k$ triangles. The computation of hyperbolic metric based on Ricci flow took 20 seconds; the computation for Fuchsian group generators and geodesic spectrum took 5 seconds. The whole pipeline is fully automatic without any human intervention.

Geodesic spectrum for shape analysis. We have done statistical analysis to study the structural difference of VS surfaces between the normal and AIS groups. 


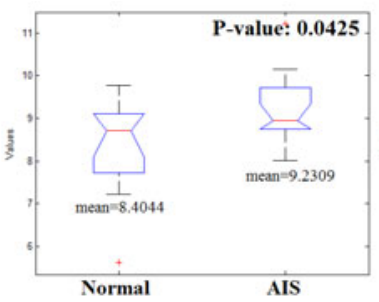

(a) box plot of $a_{1}$

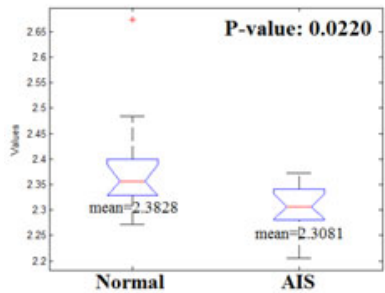

(d) box plot of $b_{1}$

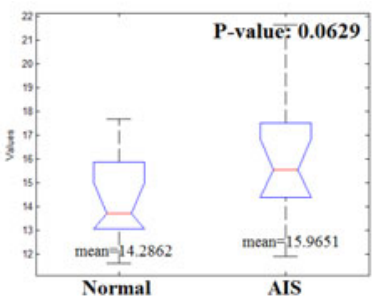

(b) box plot of $a_{2}$

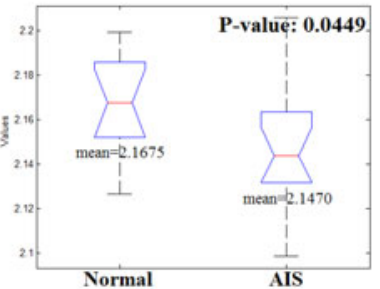

(e) box plot of $b_{2}$

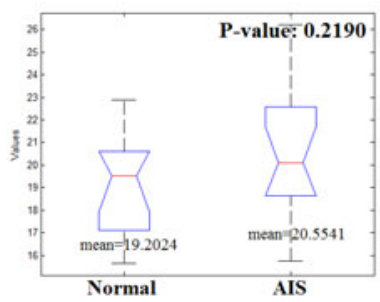

(c) box plot of $a_{3}$

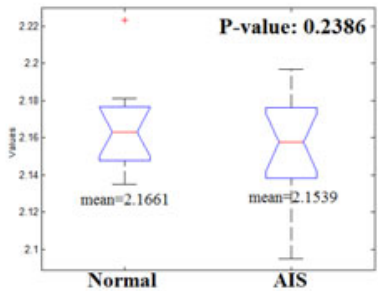

(f) box plot of $b_{3}$

Fig. 3. Distribution of geodesic spectra for the normal and AIS groups

The geodesic spectrum $\left(a_{i}, b_{i}\right)_{i=1}^{3}$ on each VS surface from the normal and AIS groups is computed. The statistical difference in the geodesic spectra between groups are evaluated using $t$ tests. The box plots of $a_{i}$ 's and $b_{i}$ 's are plotted in Figure 3. which shows the distribution of the geodesic spectra of each group. The AIS group tends to have higher $a_{i}$ 's and smaller $b_{i}$ 's. Intuitively, a higher $a_{i}$ means a longer canal whereas a smaller $b_{i}$ means a thinner canal. The shape difference in lateral canal $\left(a_{1}, b_{1}\right)$ between two groups tends to be more statistically significant with $\mathrm{P}$-value $<0.05$. In particular, the AIS group tends to have smaller $b_{1}$ and $b_{2}$ with high statistical significance. It means the lateral and superior canals are generally thinner for AIS subjects. We also studied the difference in shape index using different combination of $a_{i}$ and $b_{i}$ between groups, and their statistics are shown in Table 1. The ratio $a_{1} / b_{1}$ tends to be larger in the AIS group with high statistical significance $(\mathrm{P}=0.0338)$. It means the conformal modules of the lateral canal are significantly different. This implies once again that the shape in the lateral canal tends to be significantly different between the two groups.

Table 1. Statistics on geodesic spectra between the normal and AIS groups

\begin{tabular}{c|ccl}
\hline Combination & Mean(Normal) & Mean(AIS) & P-value \\
\hline$b_{1}+b_{2}+b_{3}$ & $6.7164 \pm 0.1103$ & $6.6091 \pm 0.0641$ & 0.0040 \\
$a_{1}+a_{2}+a_{3}$ & $41.8930 \pm 4.1591$ & $45.7500 \pm 5.5327$ & 0.0563 \\
$a_{1}+b_{1}+a_{2}+b_{2}+a_{3}+b_{3}$ & $48.6094 \pm 4.0848$ & $52.3591 \pm 5.4889$ & 0.0603 \\
$a_{1} / b_{1}$ & $3.5514 \pm 0.6145$ & $4.0070 \pm 0.4397$ & 0.0338 \\
$a_{2} / b_{2}$ & $6.5982 \pm 0.8969$ & $7.4461 \pm 1.2299$ & 0.0568 \\
$a_{3} / b_{3}$ & $8.8719 \pm 1.0674$ & $9.5539 \pm 1.5345$ & 0.2036 \\
\hline
\end{tabular}




\section{Conclusion}

We present a rigorous algorithm which computes the global feature, called the geodesic spectrum, for analyzing high-genus shapes. The key is to compute the canonical hyperbolic geodesic loops of the surface, using the Ricci flow method. The experiments on the morphometry study of the vestibular system (VS) of 12 normal and 15 AIS subjects show the effectiveness of our algorithm and reveal statistical shape difference in the VS between right-thoracic AIS and normal subjects. The performance demonstrates the great potential for AIS disease detection. In general, our proposed model is stable and can be applied to shape analysis for general anatomical structures of any genus.

\section{Acknowledgments}

Xianfeng $\mathrm{Gu}$ is supported by NIH grant 1R01EB0075300A1, NSF IIS 0916286, CCF0916235, CCF0830550, III0713145, and ONR N000140910228.

\section{References}

1. Ashburner, J., Hutton, C., Frackowiak, R., Johnsrude, I., Price, C., Friston, K., Bg, L.W.: Identifying global anatomical differences: Deformation-based morphometry. Human Brain Mapping 6(5-6), 348-357 (1998)

2. Bradshaw, A., Curthoys, I., Todd, M., Magnussen, J., Taubman, D., Aw, S., Halmagyi, G.: A mathematical model of human semicircular canal geometry: a new basis for interpreting vestibular physiology. J. Assoc. Res. Otolaryngol. 11(2), 145159 (2010)

3. Byl, N., Gray, J.: Complex balance reactions in different sensory conditions: adolescents with and without idiopathic scoliosis. J. Orthop. Res. 11(2), 215-227 (1993)

4. Chow, B., Luo, F.: Combinatorial Ricci flows on surfaces. Journal of Differential Geometry 63(1), 97-129 (2003)

5. Chung, M.K., Dalton, K.M., Davidson, R.J.: Tensor-based cortical morphometry via weighted spherical harmonic representation. IEEE Transactions on Medical Imaging 27(8), 1143-1151 (2008)

6. Dale, A.M., Fischl, B., Sereno, M.I.: Cortical surface-based analysis - I. segmentation and surface reconstruction. Neuroimage 9, 179-194 (1999)

7. Davies, R.H., Twining, C.J., Allen, P.D., Cootes, T.F., Taylor, C.J.: Shape discrimination in the hippocampus using an MDL model. In: Taylor, C.J., Noble, J.A. (eds.) IPMI 2003. LNCS, vol. 2732, pp. 38-50. Springer, Heidelberg (2003)

8. Erickson, J., Whittlesey, K.: Greedy optimal homotopy and homology generators. In: SODA 2005: Proceedings of the sixteenth annual ACM-SIAM symposium on Discrete algorithms, pp. 1038-1046 (2005)

9. Guo, X., Chau, W., Hui-Chan, C., Cheung, C., Tsang, W., Cheng, J.: Balance control in adolescents with idiopathic scoliosis and disturbed somatosensory function. Spine 31, 437-440 (2006)

10. Hamilton, R.S.: The Ricci flow on surfaces. Mathematics and general relativity 71 , 237-262 (1988)

11. Herman, R., Mixon, J., Fisher, A., Maulucci, R., Stuyck, J.: Idiopathic scoliosis and the central nervous system: a motor control problem. Spine 10(1) (1985) 
12. Jin, M., Kim, J., Luo, F., Gu, X.: Discrete surface Ricci flow. IEEE Transactions on Visualization and Computer Graphics 14(5), 1030-1043 (2008)

13. Jin, M., Zeng, W., Luo, F., Gu, X.: Computing Teichmüller shape space. IEEE Transactions on Visualization and Computer Graphics 15(3), 504-517 (2009)

14. Lambert, F.M., Malinvaud, D., Glaunés, J., Bergot, C., Straka, H., Vidal, P.-P.: Vestibular asymmetry as the cause of idiopathic scoliosis: A possible answer from Xenopus. J. Neuroscience 29, 12477-12483 (2009)

15. Pizer, S., Fritsch, D., Yushkevich, P., Johnson, V., Chaney, E.: Segmentation, registration, and measurement of shape variation via image object shape. IEEE Transaction on Medical Imaging 18(10), 851-865 (1999)

16. Reuter, M., Wolter, F.-E., Peinecke, N.: Laplace-beltrami spectra as "shape-dna" of surfaces and solids. CAD 38(4), 342-366 (2006)

17. Reuter, M., Wolter, F.-E., Shenton, M., Niethammer, M.: Laplace-beltrami eigenvalues and topological features of eigenfunctions for statistical shape analysis. CAD 41(10), 739-755 (2009)

18. Munkres, J.R.: Elements of Algebraic Topology. Addison-Wesley Co., Reading (1984)

19. Schoen, R., Yau, S.-T.: Lectures on Differential Geometry. International Press, Boston (1994)

20. Shi, L., Wang, D., Chu, W., Heng, P., Burwell, R., Cheng, J.: Statistical morphometry of the vestibular system in adolescent idiopathic scoliosis. In: 6th Annual World Congress for Brain Mapping and Image Guided Therapy. Harvard Medical School, Boston (2009)

21. Simoneau, M., Lamothe, V., Hutin, E., Mercier, P., Teasdale, N., Blouin, J.: Evidence for cognitive vestibular integration impairment in idiopathic scoliosis patients. BMC Neuroscience 10(102) (2009)

22. Simoneau, M., Richer, N., Mercier, P., Allard, P., Teasdale, N.: Sensory deprivation and balance control in idiopathic scoliosis adolescent. Experimental Brain Research 170, 576-582 (2005)

23. Zeng, W., Samaras, D., Gu, X.D.: Ricci flow for 3D shape analysis. IEEE Transactions on Pattern Analysis and Machine Intelligence 32(4), 662-677 (2010) 